\title{
TOPOLOGICAL REPRESENTATION OF ALGEBRAS. II
}

\author{
BY \\ IRVING KAPLANSKY(')
}

1. Introduction. In this paper we continue the study of algebraic algebras that was begun in $[2]\left({ }^{2}\right)$. The latter was devoted almost entirely to the commutative case, which may be described as the case where $x y-y x$ vanishes. A weaker hypothesis is that some fixed polynomial vanishes; in the terminology of [8] we assume a polynomial identity.

It turns out that many of the important features of the commutative case survive in this generalization. From one point of view this may be ascribed to the fact that the center is large enough to yield information on the structure of the algebra (a priori, the center need not have contained anything but $0)$. We cite an explicit, though rather crude result: if a semi-simple algebraic algebra with a polynomial identity is infinite-dimensional, so is its center.

In greater detail, we show that the algebras studied are built by a finite number of ring extensions out of "homogeneous" parts; and with a further countability assumption we can describe the structure of the homogeneous case. While this is by no means a complete structure theory, it is sufficiently penetrating to yield, virtually at a glance, a theorem which does not seem to be otherwise accessible: any algebraic algebra satisfying a polynomial identity is locally finite $\left({ }^{3}\right)$. As noted in [8], this contains several earlier results on local finiteness of algebraic algebras.

2. Construction of idempotents. We begin by recalling the definition of regularity, and of the two variants which were introduced in [2].

(a) A ring is regular, if for every $a$ there exists an $x$ with $a x a=a$.

(b) A ring is strongly regular if for every $a$ there exists an $x$ with $a^{2} x=a$.

(c) A ring is biregular if every principal two-sided ideal can be generated by a central idempotent.

In this paper we shall make use of still another variant introduced by McCoy [14].

(d) A ring is $\pi$-regular if for every $a$ there exists an element $x$ and an integer $n$ (depending on $a$ ) such that $a^{n} x a^{n}=a^{n}$.

The relations among these concepts may be summarized as follows: strong regularity implies biregularity and regularity, and the latter implies $\pi$-regularity.

Presented to the Society, November 26, 1949; received by the editors April 16, 1949.

(1) This paper was written while the author was a fellow of the John Simon Guggenheim Memorial Foundation, on leave from the University of Chicago.

(2) Numbers in brackets refer to the bibliography at the end of the paper.

(3) An algebra is locally finite if every finitely generated subalgebra is finite-dimensional. 
A good part of our study will be generalized by treating $\pi$-regular rings instead of algebraic algebras. This is indeed a generalization since any algebraic algebra is $\pi$-regular. To see this we note $[2$, p. 463] that if an element $a$ in an algebra satisfies a polynomial equation of degree $m$, then we may write $a^{m+1} x$ $=a^{m}$ with $x$ commuting with $a$ (in fact a polynomial in $a$ ); then $a^{m}=a^{2 m} x^{m}$ $=a^{m} x^{m} a^{m}$. Further connections between $\pi$-regularity and other conditions are noted in the appendix,

The equation $a^{n} x a^{n}=a^{n}$ implies that $a^{n} x$ is an idempotent, and moreover it is 0 only if $a^{n}=0$. This ability to construct idempotents will be of central importance in the sequel. We note the following explicitly: in a r-regular ring every non-nil right ideal contains a nonzero idempotent. It would be of interest to know how far one can get on the basis of this weaker assumption; the fact that it is not preserved under homomorphism is a notable disadvantage. On the other hand, it covers a decidedly wider class of rings, for example, all primitive rings with minimal ideals. Moreover it is a sufficient hypothesis for the following useful result.

TheOREM 2.1. Let $A$ be a ring in which every non-nil right ideal contains a nonzero idempotent. Then either $A$ contains an infinite number of orthogonal idempotents, or else it has the descending chain condition modulo its radical.

Proof. Our hypothesis implies that the radical $R$ of $A$ is a nil ideal. In view of the known device for building orthogonal idempotents in $A$ mapping on given ones in $A / R$, we can confine ourselves to the case $R=0$.

Suppose that $A$ does not have an infinite set of orthogonal idempotents. Then it is impossible for $A$ to have a properly descending chain of right ideals $\left\{e_{i} A\right\}$ generated by idempotents, for

$$
\left(1-e_{2}\right) e_{1},\left(1-e_{3}\right) e_{2} e_{1}, \cdots,\left(1-e_{n}\right) e_{n-1} \cdots e_{1}, \cdots
$$

would be a set of orthogonal nonzero idempotents. Select an idempotent $e$ such that $e A$ is minimal among right ideals generated by idempotents. Since every nonzero right ideal contains a nonzero idempotent, $e A$ will be actually minimal. The ideal $A e A$ will then be a simple ring with minimal ideals. If it violates the descending chain condition, it will have an infinite number of orthogonal idempotents (this can be easily seen from the structure theory for such rings in [3], or proved directly). So $A e A$ satisfies the chain condition, and in particular has a unit element and is a direct summand of $A$. We may continue in this fashion to tear off simple direct summands. If the process does not terminate, we get an infinite number of orthogonal idempotents.

REMARK. Theorem 2.1 has connections with three earlier results in the literature.

(a) It contains as a special case Lemma 2 of [11], which asserts that a regular ring either has the chain condition or infinitely many orthogonal idempotents. In this connection we can go further: by a slight extension of 
the argument of $[11$, Lemma 1] we can prove that a $\pi$-regular Banach algebra is finite-dimensional modulo its radical.

(b) Theorem 2.1 can be used to simplify substantially the proof of $[9$, Theorem 9]. It is evident that the hypothesis of [9, Lemma 8] rules out the possible existence of an infinite number of orthogonal idempotents. Hence parts (b) and (c) of the proof of that lemma can be suppressed.

(c) Malcev [13] has proved that a representable $\left({ }^{4}\right)$ semi-simple algebraic algebra has the descending chain condition. Since it is clear that a representable ring cannot have infinitely many orthogonal idempotents, this is a special case of Theorem 2.1 .

In the sequel we shall mainly be concerned with rings which are of bounded index in the sense that there is a fixed upper bound to the indices of nilpotent elements. If the precise upper bound in the ring $A$ is $n$, we shall say that $A$ is of index $n$. We note at this point that a semi-simple ring satisfying a polynomial identity is of bounded index; this follows from the main result in [8], and a stronger theorem is proved in [12].

The effect of the assumption of bounded index on a $\pi$-regular ring is noted in Theorem 2.3. The following is a preparatory lemma.

LEMMA 2.2. Let $B$ be a homomorphic image of the $\pi$-regular ring $A$, and suppose that $B$ contains idempotents $e, f$ with $e B \supset f B$. Suppose further that $e^{*}$ is an idempotent in $A$ such that $e^{*} A$ maps on $e B$. Then $A$ contains an idempotent $f^{*}$ such that $e^{*} A \supset f^{*} A$ and $f^{*} A$ maps on $f B$.

There is a right multiple $e^{*} w$ of $e^{*}$ that maps on $e$. Let $x$ be any element mapping on $f$ and $y=e^{*} w x$. We have $y^{n} z y^{n}=y^{n}$; pick $f^{*}=y^{n} z$. Then $e^{*} A \supset f^{*} A$; also $y^{n}$ maps on $f$, and so $f^{*}$ maps on a right multiple of $f$ of which $f$ is in turn a right multiple. That is, $f^{*} A$ maps on $f B$.

THEOREM 2.3. Let $A$ be a $\pi$-regular ring of index $n$ and $P$ a primitive ideal in $A$. Then $A / P$ is a matrix ring over a division ring, the matrices being at most $n$ by $n$.

Proof. Along with $A, A / P$ is $\pi$-regular. Using Theorem 2.1 if necessary, we see that if $B=A / P$ is not as stated, we can find in $B$ a properly descending chain $e_{1} B \supset \cdots \supset e_{n+2} B$ with $e_{i}$ idempotents (if hard pressed, we take $e_{n+2}=0$ ). By Lemma 2.2 we build idempotents $g_{1}, \cdots, g_{n+2}$ in $A$ with $g_{i} A$ properly descending and mapping on $e_{i} B$. We then pass to the orthogonal idempotents

$$
h_{1}=\left(1-g_{2}\right) g_{1}, \cdots, h_{n+1}=\left(1-g_{n+2}\right) g_{n+1} \cdots g_{1} .
$$

The $h$ 's will map on nonzero orthogonal idempotents $f_{1}, \cdots, f_{n+1}$ in $B$. Now it follows from $[5$, Lemma 4$]$ that $f_{1} B f_{2} \cdots B f_{n+1} \neq 0$. Hence there exist

(4) A ring is representable if it is isomorphic to a ring of matrices over a field. 
$a_{i}$ in $A$ such that $h_{1} a_{1} h_{2} \cdots h_{n} a_{n} h_{n+1} \neq 0$. The element $h_{1} a_{1} h_{2}+h_{2} a_{2} h_{3}+\cdots$ $+h_{n} a_{n} h_{n+1}$ is then nilpotent of index $n+1$, a contradiction.

In $\$ 4$ we shall make use of this result in the following way. Let $A$ be a $\pi$-regular ring and $X$ the set of primitive ideals $P$ such that $A / P$ is a matrix ring over a division ring with matrices of order not greater than $n$. Let $I$ denote the intersection of the ideals comprising $X$. Then it is clear that $A / I$ has index not greater than $n$. Hence it follows from Theorem 2.3 that every primitive jdeal containing $I$ lies in $X$. In other terminology: $X$ is a closed subset of the structure space of $A$.

3. Ideals and subrings. We shall later make use of the facts collected in Theorem 3.1. Actually we shall be exclusively concerned with rings for which every primitive image satisfies the chain condition, and the proof could be simplified by confining ourselves to such rings. However, for the sake of possible future application, it seems worth while to obtain the results in complete generality.

Theorem 3.1. Let $A$ be any ring and $B$ either (a) a two-sided ideal in $A$, (b) a subring of the form $e A e$, e an idempotent, or (c) a subring of the form $(1-e) A(1-e), e$ an idempotent $\left(^{5}\right)$. Then there is a one-to-one correspondence between the primitive ideals of $B$ and those primitive ideals of $A$ not containing $B$. The mapping is implemented by $P \rightarrow P \cap B, P$ primitive in $A$, and it is a homeomorphism in the topologies of the structure spaces of $A$ and $B$.

Proof. Suppose that $B$ is an ideal in $A$ and $P$ a primitive ideal in $A$ not containing $B$. Then $(P+B) / P$, being an ideal in the primitive ring $A / P$, is itself a (nonzero) primitive ring. Since $B /(P \cap B) \cong(P+B) / P, P \cap B$ is a primitive ideal in $B$. We shall now show that this mapping is (1) onto, (2) one-to-one, (3) a homeomorphism.

(1) Let $Q$ be a primitive ideal in $B$. For a suitable regular $\left(^{6}\right)$ maximal right ideal $N$ in $B, Q$ is the set of all $x$ in $B$ with $B x \subset N$. Let $g \in B$ be a left unit modulo $N$. We assert that $N+N A+(1-g) A$ is a proper right ideal in $A$. For if not, we have

$$
n+\sum n_{i} a_{i}+(1-g) a=g
$$

for suitable $n, n_{i} \in N, a, a_{i} \in A$. From this equation we first deduce $a \in B$ (since all other terms are in $B$ ). Then $(1-g) a \in N$. A right multiplication by $g$ yields $g^{2} \in N$, a contradiction. Hence $N+N A+(1-g) A$ is proper and may be expanded to a regular maximal right ideal $M$ in $A$ with $g$ as left unit. Let $P$ be the primitive ideal in $A$ consisting of all $y$ with $A y \subset M$. We shall verify

(5) If $A$ lacks a unit element, the notation $(1-e) A(1-e)$ is shorthand for the set of all $x-e x-x e-e x e$.

(8) A right ideal $M$ is regular with left unit $g$ if $g x-x$ is in $M$ for all $x$. We trust there will be no confusion between this and the other meaning of "regular." It should be noted that a proper right ideal cannot contain any power of its left unit. 
that $P \cap B=Q$. (i) Let $z \in P \cap B$. To prove $z \in Q$ we have to show $B z \subset N$. If not, the right ideal in $B$ generated by $B z$ and $N$ would be all of $B$ and would in particular contain $g$; this is impossible since $B z$ and $N$ are part of $M$. (ii) If $w \in Q$, we have $g A w \subset B w \subset N \subset M$, and hence $A w \subset M, w \in P$.

(2) Suppose $P_{1} \cap B=P_{2} \cap B$ for $P_{1}, P_{2}$ primitive in $A$. Then $P_{2} B$ maps into 0 in $A / P_{1}$. By $[5$, Lemma 4$]$ either $B \subset P_{1}$, which is excluded, or $P_{2} \subset P_{1}$. Similarly $P_{1} \subset P_{2}$ and $P_{1}=P_{2}$.

(3) In the direction $P \rightarrow P \cap B$ the continuity of the mapping is evident. To prove continuity in the reverse direction we assume that $P \cap B$ contains $\cap\left(P_{i} \cap B\right)$ and have to prove that $P$ contains $\cap P_{i}$. Evidently $P$ contains $\left(\cap P_{i}\right) B$. Since $B \subset P$ is excluded, we have $[5$, Lemma 4$] \cap P_{i} \subset P$.

(b)-(c). To treat these two cases together we write $B=f A f$ where $f$ stands respectively for $e$ and $1-e$. (The ensuing maneuvers are legal even if $f$ is not an actual ring element.) We begin with the readily verified assertion that every two-sided ideal $J$ in $B$ is of the form $J=I \cap f A f=f I f$ where $I$ is the twosided ideal in $A$ generated by $J$; and that $f A f / f I f \cong f^{*}(A / I) f^{*}$, where $f^{*}$ is the image of $f \bmod I$. Thus the mapping under discussion is $P \rightarrow f P f$. To see that $f P f$ is primitive when $P$ is, we note that if $G$ is a faithful irreducible (right) module for $A / P$, then $G f^{*}$ is a faithful irreducible module for $f^{*}(A / P) f^{*}$. As in part (a) of the proof, we proceed to verify statements (1)-(3).

(1) Let $Q, N$, and $g$ be as in part (a) above. This time we argue that $R=N+N A+(1-f) A+(1-g) A$ is a proper ideal. For suppose that $R=A$. A left and right multiplication by $f$, and the observation that $f(1-g) A f$ $=(1-g) f A f \subset N$, show that $N$ contains $f A f$, a contradiction. Thus $R$ can be expanded to a regular maximal right ideal $M$; we let $P$ denote the primitive ideal attached to it, and shall prove $Q=f P f=P \cap f A f$. To check the inclusion $Q \subset P$ we have to show $A Q \subset M$, and this follows from $g A Q \subset B Q \subset N \subset M$. To prove the reverse inclusion $f P f \subset Q$, we must verify $B f P f \subset N$. Now $B f P f \subset f A P \subset f M \subset M$ (the last inclusion is a consequence of the fact that $M$ contains $(1-f) A)$. Hence $B f P f$ must be contained in $N$, for otherwise the right ideal in $B$ it generates with $N$ would contain all of $B$ and in particular $g$, contrary to the fact that $B f P f$ and $N$ are both in $M$.

(2) Let $C$ denote the two-sided ideal in $A$ generated by $f A$. If $f P_{1} f=f P_{2} f$, then $P_{1}$ contains $C P_{2} C$, and hence $P_{1} \supset P_{2}$, since $P_{1} \supset C$ is excluded. The proof of (3) in (a) can similarly be adapted by using $C$ in place of $B$.

The proof of the following lemma is left to the reader.

Lemma 3.2. If $A$ is a $\pi$-regular ring, so is any two-sided ideal in $A$ and any subring of the form $e A e$ or $(1-e) A(1-e)$, e an idempotent.

4. Reduction to strongly regular rings. Let $A$ be a $\pi$-regular ring and let $I_{n}$ denote the intersection of the primitive ideals $M$ such that $A / M$ is an $r$ by $r$ matrix ring over a division ring, $r \leqq n$ (these ideals will of course also be regular maximal). From Theorems 2.3 and 3.1 it is readily deduced that all prim- 
itive images of the ring $I_{n-1} / I_{n}$ are precisely $n$ by $n$ matrix rings over division rings. Moreover, $I_{n-1} / I_{n}$ is semi-simple. If $A$ is semi-simple and of bounded index, the chain of $I$ 's will reach 0 in a finite number of steps (Theorem 2.3). It follows that a semi-simple $\pi$-regular ring of bounded index is built, by a finite number of ring extensions, out of rings that are homogeneous in the following sense.

Definition. A $\pi$-regular ring is homogeneous if it is semi-simple and such that for every primitive ideal $P, A / P$ is an $n$ by $n$ matrix ring over a division ring ( $n$ independent of $P$ ).

Any strongly regular ring is homogeneous (with $n=1$ ), and conversely a homogeneous $\pi$-regular ring with $n=1$ is strongly regular. In the succeeding theorems we shall see that a homogeneous $\pi$-regular ring is not much more general than a matrix ring over a strongly regular ring.

THEOREM 4.1. Let $A$ be a homogeneous $\pi$-regular ring. Then its structure space $S$ is locally compact and zero-dimensional, and $A$ contains the characteristic function of any compact open set in $S\left({ }^{7}\right)$.

Proof. The theorem is known from [2] for $n=1$, and we proceed by induction on $n$. Let $M, N$ be distinct points (=maximal ideals) in $S$. There exists an element $b$ lying in $N$ and mapping on a primitive idempotent $\bmod M$. Write $e=b^{r} x$, where $b^{r} x b^{r}=b^{r}$. Then $e$ is an idempotent, and again lies in $N$ and maps on a primitive idempotent $\bmod M$. Let us introduce three subsets of $S$ : (1) $X$, the set where $e$ maps on 0 , (2) $Y$, the set where $e$ maps on 1 , (3) $Z$, the set where $e$ maps on a primitive idempotent. We note that these sets are disjoint and that $X$ contains $N$ and $Z$ contains $M$; except for $n=2$, they presumably do not exhaust $S$. By virtual definition of the topology, $X$ and $Y$ are closed. By Theorem 3.1, the structure space of the ring $B=(1-e)$ $A(1-e)$ is in a natural way homeomorphic to the complement $Y^{\prime}$ of $Y$; let us simply identify it with $Y^{\prime}$. Then $X$ becomes the set of regular maximal ideals of $B$ modulo which we get $n$ by $n$ matrices. By the same token, the complement of $X$ in $Y^{\prime}$ is the set of ideals modulo which the matrices are smaller than $n$ by $n$. By Theorem 2.3, this latter set is closed in $Y^{\prime}$. Hence $X$ is open in $Y^{\prime}$, and since $Y^{\prime}$ is open in $S, X$ is open in $S$ (as well as closed). Next we note (Theorem 3.1 again) that the structure space of $e A e$ is homeomorphic to the complement $X^{\prime}$ of $X$. Since $e A e$ has a unit element, $X^{\prime}$ is compact. We have thus constructed a compact open and closed set (namely $X^{\prime}$ ) which contains $M$ but not $N$. This proves that $S$ is locally compact and zero-dimensional.

It remains to prove the last statement of the theorem. For this purpose we introduce next $C$, the intersection of the ideals comprising $X$, and $D=(1-e)$ $C(1-e)$. The structure space of $D$ is the complement (say $W$ ) of $X \cup Y$, and

(7) We are following the terminology of [2] in the use of the terms "zero-dimensional" and "characteristic function." 
the subset $Z$ of $W$ constitutes precisely those ideals of $D$ modulo which we get $(n-1)$ by $(n-1)$ matrices; moreover these are the largest matrices that occur in the simple images of $D$. It follows (as in the preceding paragraph) that $Z$ is open in $W$ and hence in $S$.

We are now ready for the final construction. Let $F$ denote the intersection of the ideals comprising the closed set $Z^{\prime}=$ complement of $Z$; the structure space of $F$ is $Z$, and the rings $e F e$ and $(1-e) F(1-e)$ likewise have $Z$ for their structure space. The presence of a unit in $e F e$ shows that $Z$ is compact. Now by Lemma $2.3,(1-e) F(1-e)$ is again $\pi$-regular, and moreover it is homogeneous of index $n-1$. Its structure space being compact, it will have a unit element $f$ by induction. It is clear that $e+f$ is a unit element in $A$ modulo every ideal of $Z$. Thus the point $M$ has a neighborhood (namely $Z$ ) such that $A$ has a simultaneous unit element modulo all the ideals of the neighborhood. We have now verified the hypothesis of $[2$, Lemma 1.4$]$ and we have the desired conclusion that $A$ contains a characteristic function of every compact open set in $S$. This concludes the proof of Theorem 4.1 .

THEOREM 4.2. A homogeneous $\pi$-regular ring with unit element is a matrix ring over a strongly regular ring.

Proof. Given a point $M$ in the structure space of our ring $A$, we pick an idempotent mapping into a primitive idempotent there. As noted in the proof of Theorem 4.1, it will continue to map into a primitive idempotent in a neighborhood of $M$. Then Theorem 4.1 and a standard argument of [2] (see particularly footnote 7 ) allow us to build an element $e_{11}$ mapping on a primitive idempotent everywhere. By induction on the index, the ring $\left(1-e_{11}\right)$ $A\left(1-e_{11}\right)$ is a matrix ring, say with matrix units $e_{i j}(2 \leqq i, j \leqq n)$. We proceed to construct the still missing matrix units. At a given point $M$ we pick $x, y$ such that $x y, y x$ agree with $e_{11}, e_{22}$ respectively, $\bmod M$. We write $u=e_{11} x e_{22}$, $v=e_{22} y e_{11}$; then $u v, v u$ still agree with $e_{11}, e_{22} \bmod M$. In the strongly regular ring $e_{11} A e_{11}$ we observe that $u v$ maps on the unit $e_{11}$ at the point corresponding to $M$; hence it continues to map on $e_{11}$ in a neighborhood of $M$. Likewise $v u$ maps on $e_{22}$ in a neighborhood of $M$. The standard argument enables us to build elements $e_{12}, e_{21}$ with $e_{12} e_{21}=e_{11}, e_{21} e_{12}=e_{22}$. Define $e_{1 i}=e_{12} e_{2 i}, e_{i 1}=e_{i 2} e_{21}$, and it is readily verified that we have a set of matrix units. Let $B$ be the subring of $A$ commuting with all the $e_{i j}$ 's. Then it is known [1, p. 19] that $A$ is isomorphic to the ring of all $n$ by $n$ matrices over $B$. That $B$ is strongly regular may be seen as follows. For $b \in B$ we have $b^{r} z b^{r}=b^{r}$. Modulo any maximal ideal $M, b$ maps into a scalar matrix $b^{*}$; either $b^{*}=0$ or $b^{*}$ is a nonsingular scalar matrix, and in either case $b^{2} z \equiv b \bmod M$.

REMARK. The preceding theorem has shown that a decisive result is available for homogeneous rings if there is a unit. But it should be noted that in analyzing a nonhomogeneous $\pi$-regular ring, there is virtually nothing to be gained by assuming a unit element; the "homogeneous parts" will in any 
event lack unit elements in general. Thus something must be done without the assumption of a unit, and the next theorem shows that a countability restriction leads to a satisfactory result.

THEOREM 4.3. Let $A$ be a homogeneous $\pi$-regular ring, with center $Z$. Then $A / A Z$ is a nil-ring and $A Z$ is regular and biregular. If $A Z$ is countably generated, it is the direct sum of a countable number of rings with unit elements, and so is a matrix ring over a strongly regular ring.

Proof. First we prove that any idempotent $e$ is in $A Z$. The set $X$ of regular maximal ideals not containing $e$ coincides with the structure space of $e A e$ (Theorem 3.1); the latter is compact since $e A e$ has a unit. By Theorem 4.1, $A$ contains the characteristic function $f$ of $X$. Then $f \in Z$ and $e=e f \in A Z$.

Next, if $a$ is any element of $A$, then $a^{r} x a^{r}=a^{r}, a^{r} x$ is in $A Z$, and so is $a^{r}$. Hence $A / A Z$ is a nil-ring (of index at most that of $A$ ).

The ring $Z$ is itself $\pi$-regular [14, Theorem 1]. Since $A$ is semi-simple, $Z$ has no nilpotent elements. Hence $Z$ is actually regular. It is then known that for any finite number of elements of $Z$ there is an idempotent acting as unit element. Hence for every element of $A Z$ there is a central idempotent $g$ acting as unit. The ideal $B=A g$ is a direct summand of $A$, and since $B$ has a unit, its structure is given by Theorem 4.2. It is then clear that $B$-and hence $A Z$ is both regular and biregular.

Finally, suppose $A Z$ is countably generated. It follows from [2, Lemma 8.1] that its structure space decomposes into a countable number of disjoint open compact sets. This says that $A Z$ is the direct sum of a countable number of rings with unit. Each summand is a matrix ring by Theorem 4.2, and so is all of $A Z$.

REMARKs. 1 . Without the countability assumption it need not be true that $A Z$ is a matrix ring over a strongly regular ring. A counter-example can be constructed on the same lines as the fundamental counter-example in [2].

2. The "fragment of radical" showing up in Theorem 4.3 can really occur. An example is the ring of all sequences of 2 by 2 matrices which are ultimately constant and of the form

$$
\left(\begin{array}{ll}
0 & a \\
0 & 0
\end{array}\right) .
$$

A suitable hypothesis stronger than semi-simplicity can, however, abolish these traces of radical, as is shown in the following theorem, for example.

THEOREM 4.4. Let $A$ be a $\pi$-regular ring of bounded index such that $A$ and all its homomorphic images are semi-simple. Then $A$ is regular.

Proof. This is clear from Theorem 4.3 in the homogeneous case, and the extension to the nonhomogeneous case merely requires the following remark: if $I$ and $A / I$ are regular, so is $A$. 
In Theorem 4.4 we cannot conclude that $A$ is biregular: a counter-example is the ring of all sequences of matrices which are ultimately diagonal. In this ring the structure space is not Hausdorff. This simple example incidentally illustrates how the different homogeneous parts can combine.

We shall conclude this section with the result referred to in the introduction concerning the size of the center. Since we prefer to delay as long as possible the introduction of a field of scalars, we phrase the theorem as follows: in a semi-simple $\pi$-regular ring of bounded index, either the descending chain condition holds, or there are infinitely many central idempotents. This is clear from Theorem 4.3 in the homogeneous case. The general case follows from the following observation: let $I$ be an ideal in a ring $A$ with the property that no element of $I$ left-annihilates $I$; then the center of $I$ is contained in the center of $A$. For if $z$ is in the center of $I$, then for any $a, z a-a z$ annihilates $I$.

5. Strongly regular algebras. Having substantially accomplished a reduction to the case of a strongly regular ring $A$, we now turn our attention to the structure of the latter. We have that each $A / M$ is a division ring, and it is natural to proceed by classifying $M$ according to the order of $A / M$ over its center. Let $X_{n}$ be the set of all $M$ such that $A / M$ is of order not greater than $n^{2}$ over its center, and let $I_{n}$ be the intersection of these ideals. Then: $X_{n}$ is a closed subset of the structure space. $\operatorname{For}\left(^{8}\right) A / I_{n}$ satisfies the polynomial identity that characterizes division rings of order not greater than $n^{2}$ over their center, and hence so will $A / M$ for any $M$ containing $I_{n}$. We note next (Theorem 3.1) that in $I_{n-1} / I_{n}$ we have achieved the homogeneity appropriate in the present context: uniform order over the center at all maximal ideals. If $A$ itself satisfies a polynomial identity, the chain of $I$ 's will be finite. As in Theorem 4.3 , by a countability assumption we can reduce to the case where there is a unit element. At this point, one might hope to effect a complete reduction to the commutative case, but I have been able to do this only when $A$ is an algebra, and indeed an algebraic algebra.

Theorem 5.1. Let $A$ be an algebraic algebra over a field $F$. Suppose that $A$ has a unit element; that it is strongly regular (it is equivalent to assume that there are no nilpotent elements); and that for every maximal ideal $M$, the order of $A / M$ over its center is $n^{2}$, with $n$ independent of $M$. Then $A$ is the direct sum of a finite number of algebras, each of which is the Kronecker product, over some extension field of $F$, of a commutative algebra and a central division algebra of order $n^{2}$.

Proof. The proof is similar to that of [2, Theorem 5.2], and indeed the latter is essentially a special case of the present theorem. At a given maximal

$\left.{ }^{8}\right)$ That there is such an identity follows from the results in $\$ 2$ of [10]; in the notation used there, the identity in question is $\left[x_{1}, \cdots, x_{r(n)}\right]=0$. In a paper to be published in the Bull. Amer. Math. Soc., Amitsur and Levitzki prove that $r(n)=2 n$. 
ideal $M$, let the algebra $B=A / M$ have center $Z,[B: Z]=n^{2}$. We pick a basis $u_{i}$ for $B / Z$ and we have the multiplication table $u_{i} u_{j}=\sum c_{i j k} u_{k}, c_{i j k} \in Z$. The elements $c_{i j k}$ generate a finite-dimensional extension $K$ of $F$, and the $u$ 's and $c$ 's generate a subalgebra $C$ which is a central division algebra of order $n^{2}$ over $K$. Let us augment the $u_{\text {'s }}$ to a basis $v_{i}$ of $C / F$ with table $v_{i} v_{j}=\sum d_{i j k} v_{k}$, $d_{i j k} \in F$, and let $w_{i}$ be elements of $A$ mapping on $v_{i}$. Then the equations $w_{i} w_{j}=\sum d_{i j k} w_{k}$ will persist in a suitable open and closed neighborhood $U$ of $M$. A finite number of $U$ 's will account for all of $A$, and, by the usual use of the characteristic functions, we can reduce to the case where $U$ is the entire structure space of $A$. To simplify notation we suppose that this has already been done. Then $w_{i} w_{j}$ will actually equal $\sum d_{i j k} w_{k}$, and the $w^{\prime}$ s will generate a sub-division-algebra $C^{*}$ of $A$ isomorphic to $C$, with center $K^{*}$ isomorphic to $K$.

Let us now see what happens modulo some other maximal ideal $M_{1}$. There $C^{*}$ and $K^{*}$ will map say into $C_{1}$ and $K_{1}$. Write $B_{1}$ for $A / M_{1}$ and $Z_{1}$ for its center. Then the subalgebra $C_{1} Z_{1}$ of $B_{1}$ is the Kronecker product over $K_{1}$ of $C_{1}$ and $Z_{1}$. We now use our hypothesis that $\left[B_{1}: Z_{1}\right]=\left[C_{1}: K_{1}\right]$ to conclude that $C_{1} Z_{1}$ is all of $B_{1}$. This shows in particular that $K_{1}$ is part of $Z_{1}$. Hence $K^{*}$ is in the center of $A$-because this is true modulo every maximal ideal. We can now regard $A$ as an algebra over $K^{*}$, and $C^{*}$ becomes a central division subalgebra. It is known $\left({ }^{9}\right)$ that $A$ is the Kronecker product, over $K^{*}$, of $C^{*}$ and the commutator $D^{*}$ of $C^{*}$. That $D^{*}$ is commutative can be seen by verifying it modulo every maximal ideal ( $D^{*}$ is in fact the center of $A$ ). This completes the proof of Theorem 5.1.

6. Kurosch's problem. In analogy with Burnside's problem for groups, Kurosch has asked whether every algebraic algebra is locally finite. The previous results on this question (cf. [8] and the references given there) are extended in the following theorem.

Theorem 6.1. Any algebraic algebra satisfying a polynomial identity is locally finite.

Proof. The proof requires not much more than an inspection of the results obtained in the two preceding sections. Let $A$ be an algebraic algebra with a polynomial identity. Since any finitely generated algebra is of countable order, we may fortunately confine ourselves to the case where $A$ is of countable order. We shall make systematic use of [6, Theorem 15] which asserts that if $I$ and $A / I$ are locally finite, so is $A$. As a first application, we combine this with $[8$, Theorem 5] to reduce to the case where $A$ is semi-simple. Now $A$ is of bounded index (and of course $\pi$-regular). Following the discussion in $\$ 4$, we reduce forthwith to the case where $A$ is homogeneous in the sense of the

${ }^{(9)}[1$, Theorem 4.6]. The theorem in question states that if $B$ is a finite-dimensional central simple subalgebra of $A$, then $A$ is the Kronecker product of $B$ and its commutator. The proof given by Albert works for infinite-dimensional $A$. 
definition in $\$ 4$. Theorem 4.3 then becomes available and (in its notation) we reduce the problem to $A Z$ by another application of [8, Theorem 5]. Since $A Z$ is a matrix algebra over a strongly regular algebra, our problem has now reached the strongly regular case. We pass next to algebras which are homogeneous in the sense required for Theorem 5.1 ; because of countability we can get hold of a unit element, and we may therefore apply that theorem. Since commutative algebraic algebras are locally finite and since it is obvious that the Kronecker product of locally finite algebras is locally finite, the proof of Theorem 6.1 is thereby complete.

REMARK. For possible future use, we observe that our results also prove the following: if Kurosch's problem has an affirmative answer for algebraic algebras without nilpotent elements, then it has an affirmative answer for algebraic algebras of bounded index.

7. An approximation theorem. The results thus far obtained fall short of a reasonably complete structure theory by virtue of the failure to discuss the combination of the homogeneous parts, and the structure of the homogeneous parts in the uncountable case. As a first step in filling these gaps, it appears to be desirable to have a theorem of the Weierstrass-Stone type in order to know what functions do arise once a functional representation is achieved. Theorem 7.2 is such a result. For convenience in the discussion we introduce a definition.

Definition. Let $A$ be a ring, $B$ a subring. $B$ will be said to bi-approximate $A$ if for any element $a$ and regular maximal ideals $M, N$ in $A$, there is an element $b \in B$ such that $b \equiv a(\bmod M \cap N)$.

Lemma 7.1. If $A$ is biregular, then any bi-approximating subring $B$ is all of $A$.

Proof. The proof is based on the same idea as that of [7, Theorem 32]. Let $S$ be the structure space of $A$. (1) If $K$ is a compact subset of $S$, and $M \in S$ is not in $K$, then $B$ contains an element vanishing on $K$ and mapping on 1 at $M$. For if $N \in K$, then $B$ contains an element $b$ with $b(N)=0, b(M)=1$. The element $b$ will continue to vanish in a neighborhood of $N$. The product of a finite number of such $b$ 's gives us the desired element. (2) By multiplying by a suitable element of $B$ we may further assert that $B$ contains an element vanishing on $K$ and taking an arbitrary value at $M$. (3) For any $M \in S, B$ contains the characteristic function of a suitable neighborhood of $M$. For let $b \in B$ be any element with $b(M)=1$. There will be a compact neighborhood $K$ of $M$ with $b(K)=1$. Let $L$ denote the (compact open) set where $b$ does not vanish. By part (2), $B$ contains an element $c$ which vanishes on $K$ and at a given point $N$ in $L-K$ takes the same value as $b$. Then $b(b-c)$ is still 1 on $K$ and vanishes in a neighborhood of $N$. A finite number of these neighborhoods cover the compact set $L-K$. The product of the corresponding elements $b(b-c)$ is the characteristic function of $K$. (4) If $e, f$ are characteristic functions of sets, then $e+f-e f$ is the characteristic function of the union 
of the two sets. It follows from (3) that $B$ contains the characteristic function of any compact open set. (5) At any given point, $B$ contains an element agreeing with a given element $a \in A$. It continues to agree in a neighborhood. Use of the characteristic functions, which we know to be in $B$ by (4), allows us to build in $B$ an element agreeing everywhere with $a$.

We leave to the reader the deduction of Theorem 7.2 from Lemma 7.1.

THEOREм 7.2. If $A$ is a regular $\left({ }^{10}\right)$ ring of bounded index, then any bi-approximating subring is all of $A$.

We shall now briefly sketch how Theorem 7.2 can be used to set up a structure theory, under the further restrictive assumption that all $A / M$ are finite with a fixed upper bound. It amounts to the same thing to assume a polynomial identity in one variable. Let then $A$ be a regular $\left({ }^{10}\right)$ ring satisfying a polynomial identity in one variable, and for simplicity let us assume that $A$ has a unit. Let $G$ be a finite simple ring, large enough so that every $A / M$ is isomorphic to a subring of $G$. Let $X$ denote the space of all homomorphisms of $A$ into $G$, topologized by point-wise convergence. Then $X$ is compact and zero-dimensional, and the elements of $A$ are naturally represented as continuous functions from $X$ to $G$. There remains the question: what functions do arise? Theorem 7.2 in effect asserts that it is enough to know what we can do at pairs of points. In other words, to construct all possible $A$ 's we must name at every pair of points a semi-simple subring of $G \oplus G$; this list (there are of course compatibility conditions to be respected) determines $A$.

This is a rather unpleasantly complicated result, but it does not appear to be possible to simplify it substantially. We remark though that if we add a homogeneity assumption (for example that all $A / M$ are isomorphic), then the result can be simplified to read: $A$ is determined by the way the automorphisms of $G$ induce certain homeomorphisms of $X$.

If we attempt to imitate the foregoing procedure without the assumption of a polynomial identity in one variable-in other words, with infinite $G$ we run into the obstacle that $X$ need no longer be compact. Whether there is always a suitable subspace of $X$ which is compact is a question the author has been unable to answer.

8. Appendix. This appendix is devoted to several remarks on chain conditions. We have observed that if $a$ is an algebraic element in an algebra, then there exists $n$ and $x$ with $a^{n+1} x=a^{n}$. Now the latter condition is equivalent to the fact that the chain $(a)_{r} \supset\left(a^{2}\right)_{r} \supset \cdots$ terminates $\left({ }^{11}\right)$. Thus in an algebraic algebra there is automatically a weak chain condition present.

(10) The restriction to regular rings is made to avoid the fragments of nil-rings that show up in Theorem 4.3. It is possible to cope with this difficulty, but the added complication does not seem worth while.

(11) The notation $(a)_{r}$ stands for the principal right ideal generated by $a$ : the set of all $a x+n a$ where $x$ is a ring element and $n$ an integer. 
Since it is customary in modern algebra to use chain conditions as a fundamental hypothesis, it is natural to ask what can be deduced from just the assumption $a^{n+1} x=a^{n}$. For example, does it enable one to construct idempotents? I am unable to answer this, but add four related remarks.

(1) If it is assumed that $x$ commutes with $a$, we get $\pi$-regularity.

(2) If $n$ is independent of $a$, it can be shown that $x$ can be replaced by an $x^{\prime}$ commuting with $a$ (one uses [2, Theorem 3.1], and the fact that a matrix over a division ring is the direct sum of a nilpotent matrix and a non-singular matrix).

(3) The assumption of no nilpotent elements leads to strong regularity. This was proved by Gertschikoff [4].

(4) If we strengthen the chain condition to include all principal right ideals (instead of just descending chains of powers of a single element), we can prove a decisive result. From Theorem 8.1 we can incidentally readily deduce Gertschikoff's main theorem: if $A$ satisfies the descending chain condition on principal right ideals and has no nilpotent elements, then $A$ is the direct sum of division rings.

THEOREM 8.1. If a semi-simple ring satisfies the descending chain condition on principal right ideals, then it is a direct sum of simple rings with minimal ideals.

Proof. Take first the case of a primitive ring $A$. It has a minimal right ideal (the concept of minimal right ideal does not change if you are dealing only with principal right ideals). We take a representation of $A$ as a dense ring of linear transformations, and our task is to prove that these linear transformations have finite-dimensional range [5, Theorem 30]. Suppose on the contrary that $r$ does not. Define $a_{1}=r$, and having defined $a_{n-1}$ (which we assume has infinite-dimensional range), let $x$ be a vector not annihilated by $a_{n-1}$. Choose a linear transformation $b$ in $A$ with finite-dimensional range sending $x a_{n-1}$ into $-x a_{n-1}$, and define $a_{n}=a_{n-1}(1+b)$. Then the chain $\left(a_{n}\right)_{r}$ is properly descending, since $a_{n}$ annihilates $x$ while $a_{n-1}$ does not.

In the general case of semi-simple $A$, our hypothesis is inherited under homomorphism, and so for every primitive ideal $P, A / P$ is simple with minimal ideals. Our task here is to prove that every element vanishes at all but a finite number of the coordinates of the subdirect sum of $\left\{A / P_{i}\right\}$. Suppose that $r$ is nonzero at an infinite number of coordinates, set $a_{1}=r$, and suppose $a_{n-1}$ defined. Take two of the places where $a_{n-1}$ is nonzero, and let $b$ be an element vanishing at one of them and acting as a right unit for $a_{n-1}$ at the other. If $a_{n-1} b$ is still nonzero at an infinite number of coordinates, we set $a_{n}=a_{n-1} b$; otherwise $a_{n}=a_{n-1}(1-b)$. We thus build a strictly descending $\operatorname{chain}\left(a_{n}\right)_{r}$. 


\section{BIBLIOGRAPHY}

1. A. A. Albert, Structure of algebras, Amer. Math. Soc. Colloquium Publications, vol. 24, New York, 1939.

2. R. Arens and I. Kaplansky, Topological representation of algebras, Trans. Amer. Math. Soc. vol. 63 (1948) pp. 457-481.

3. J. Dieudonné, Sur le socle d'un anneau et les anneaux simples infinis, Bull. Soc. Math. France vol. 70 (1942) pp. 46-75.

4. A. Gertschikoff, Über Ringe, die in eine direkte Summe von Körpern zerlegbar sind. Mat. Sbornik N. S. vol. 7 (1940) pp. 591-597 (Russian with German summary).

5. N. Jacobson, The radical and semi-simplicity for arbitrary rings, Amer. J. Math. vol. 67 (1945) pp. 300-320.

6. - Structure theory for algebraic algebras of bounded degree, Ann. of Math. vol. 46 (1945) pp. 695-707.

7. I. Kaplansky, Topological rings, Amer. J. Math. vol. 69 (1947) pp. 153-183. 580.

8. - Rings with a polynomial identity, Bull. Amer. Math. Soc. vol. 54 (1948) pp. 575-

9. - Topological methods in valuation theory, Duke Math. J. vol. 14 (1947) pp. 527541.

10. - Groups with representations of bounded degree, Canadian Journal of Mathematics vol. 1 (1949) pp. 105-112.

11. - Regular Banach algebras, J. Indian Math. Soc. vol. 12 (1949) pp. 57-62.

12. J. Levitzki, $A$ theorem on polynomial identities, to be published in Bull. Amer. Math. Soc.

13. A. Malcev, On the representations of infinite algebras, Mat. Sbornik N. S. vol. 13 (1943) pp. 263-286 (Russian with English summary).

14. N. McCoy, Generalized regular rings, Bull. Amer. Math. Soc. vol. 45 (1939) pp. 175-178.

Institute for Advanced Study,

Princeton, N. J. 